\title{
PERIODIC PROPERTIES OF THE SEMI-PERMANENT ATMOSPHERIC PRESSURE SYSTEMS*
}

\author{
BY \\ H. J. STEWART \\ California Institute of Technology
}

The outstanding features of the general circulation of the atmosphere are the belts of westerly winds and, on the equatorial side of these, the system of semipermanent sub-tropical high pressure areas. In a previous paper ${ }^{1}$ the author has discussed the problem of the formation of such high pressure systems. In particular, it was shown that these systems probably represent dynamically stable concentrations of vorticity similar to the Kármán "vortex street" which is formed behind any twodimensional bluff body over a wide range of values of the Reynolds number. It now appears that a further examination of the periods of the characteristic oscillations of such systems is of considerable interest. It is seen that the period of these oscillations is of the order of magnitude of years. This indicates that oscillations of this type may be of importance in the calculation of the long period displacements of the Pacific or Azores high pressure systems.

It is believed that this is the first time that atmospheric motions have been discussed which have a period of the order of magnitude of, but different from, a year. Since the weather shows large variations from one year to another, it is apparent that such motions must exist; and, since the non-seasonal variation of the only external parameter, the solar energy input, is very small, these long period motions must be explainable in terms of the free oscillations of the earth's atmosphere.

It seems that the horizontal field of motion is of primary importance in determining the motion of these large scale systems; so it is assumed that the atmosphere can be treated as a single layer of fluid of constant density with the vertical velocities being of small importance so that the pressure can be determined from the hydrostatic equation. It is also assumed that the apparent acceleration is negligible when compared to the Coriolis acceleration. In addition the effects of friction and of the variation of the Coriolis parameter with latitude are neglected. This latter factor means that the fluid motions considered are those taking place on a rotating disc rather than on a rotating sphere.

The notation used in the discussion is as follows:

$x, y=$ Cartesian coordinates on a rotating disc, $u=$ velocity in $x$ direction, $v=$ velocity in $y$ direction, $\omega=$ angular velocity of the disc, $h=$ depth of the fluid, $g=$ acceleration due to gravity.

If the motion could have been started from rest with a uniform depth $h_{0}$, the principle of conservation of the absolute vorticity states that

$$
\frac{\partial v}{\partial x}-\frac{\partial u}{\partial y}=\frac{2 \omega}{h_{0}}\left(h-h_{0}\right) .
$$

* Received August 4, 1943.

1 Stewart, H. J., Proc. Nat. Acad. of Sci., 26, 604 (1940). 
If the velocity components are eliminated from this equation by means of the geostrophic wind equations,

$$
-2 \omega v=-g \frac{\partial h}{\partial x}, \quad 2 \omega u=-g \frac{\partial h}{\partial y},
$$

an expression determining the depth of the atmosphere (i.e. the sea-level pressure) is obtained. This is

$$
\frac{\partial^{2} h}{\partial x^{2}}+\frac{\partial^{2} h}{\partial y^{2}}-\frac{4 \omega^{2}}{g h_{0}}\left(h-h_{0}\right)=0 .
$$

This equation can be further simplified by the introduction of dimensionless variables, $X=2 \omega x / \sqrt{g h_{0}}, \quad Y=2 \omega y / \sqrt{g h_{0}}$ and $\eta=\left(h-h_{0}\right) / h_{0}$. With these new variables, Eq. (3) becomes

$$
\frac{\partial^{2} \eta}{\partial X^{2}}+\frac{\partial^{2} \eta}{\partial Y^{2}}-\eta=0
$$

In terms of the dimensionless depth and dimensionless velocities defined by $U=u / \sqrt{g h_{0}}$ and $V=v / \sqrt{g h_{0}}$, the geostrophic wind equation can be rewritten as

$$
\frac{\partial \eta}{\partial X}=V, \quad \frac{\partial \eta}{\partial Y}=-U
$$

The only solution of Eq. (4) which vanishes at infinity and which represents flow in circles about the origin and thus corresponds to a simple vortex is

$$
\eta=\alpha K_{0}(r)
$$

where $\alpha$ is an arbitrary constant, $r=\sqrt{X^{2}+Y^{2}}$ and $K_{0}(r)$ is a modified Bessel function ${ }^{2}$ of the second kind. If $\alpha$ is positive the motion is anticyclonic; if $\alpha$ is negative the motion is cyclonic. All of the motions considered in the present investigation are built up through superposition of vortices of this type. From Eq. (5), this vortex has a dimensionless tangential velocity, $u_{\theta}$, given by

$$
u_{\theta}=-\alpha K_{1}(r)=\frac{\partial \eta}{\partial r} .
$$

The geostrophic wind equations used in the above development can be shown to be valid unless $r \ll 1$.

Based on a homogeneous atmosphere having a mean sea-level pressure and density of $1.013 \times 10^{6}$ dynes $/ \mathrm{cm}^{2}$ and $1.22 \times 10^{-3} \mathrm{gm} / \mathrm{cm}^{3}$ respectively, the same as the standard atmosphere, the characteristic velocities and distances used above to produce dimensionless variables are $\sqrt{g h_{0}}=2.87 \times 10^{4} \mathrm{~cm} / \mathrm{sec}$ and $\sqrt{g h_{0}} / 2 \omega=1.97 \times 10^{8} \mathrm{~cm}$. At a distance of $2000 \mathrm{~km}$. from the center of the Pacific or Azores high pressure systems, the characteristic velocity is of the order of 10 meters/second. Since $K_{1}(1)=0.602$, this indicates that these anticyclones have a strength such that $\alpha$ is approximately 0.06 .

If the interaction between the northern and southern hemispheres is neglected, the ring of subtropical anticyclones can be roughly represented by $N$ equal anticyclones of the type given by Eq. (5) which are placed on a ring of radius $a$ and spaced

\footnotetext{
${ }^{2}$ Grey, Mathews and MacRobert, Bessel Functions, Macmillan and Co., London, 1931.
} 
at equal angles $\tau=2 \pi / N$ as shown in Fig. 1. In the northern hemisphere, the Pacific and Azores high pressure regions are well defined. They are about $120^{\circ}$ of longitude apart. There is some evidence of a third such system over India and equidistant from the other two; however this evidence is far from conclusive due to the low level interference from the monsoon. In the southern hemisphere there are also three such systems. The best model is thus obtained with $N=3$.

The surface deflection for such a system in its equilibrium state is

$$
\eta=\alpha \sum_{n=1}^{N} K_{0}\left[a^{2}+r^{2}-2 a r \cos (\theta-n \tau)\right]^{1 / 2} .
$$

From Eq. 5 the dimensionless velocities in the radial and tangential directions, $u_{r}$ and $u_{\theta}$ respectively, are given by

$$
u_{r}=-\frac{1}{r} \frac{\partial \eta}{\partial \theta}, \quad u_{\theta}=\frac{\partial \eta}{\partial r} .
$$

The velocity of any vortex is the velocity at that point due to all of the remaining vortices. From the second of the expressions of Eq. 9, the system shown in Fig. 1 is seen to have a dimensionless angular velocity $\Omega$ given by

$$
\Omega=-\frac{\alpha}{a} \sum_{n=1}^{N-1} K_{1}\left[2 a \sin \frac{1}{2} n \tau\right] \sin \frac{1}{2} n \tau .
$$

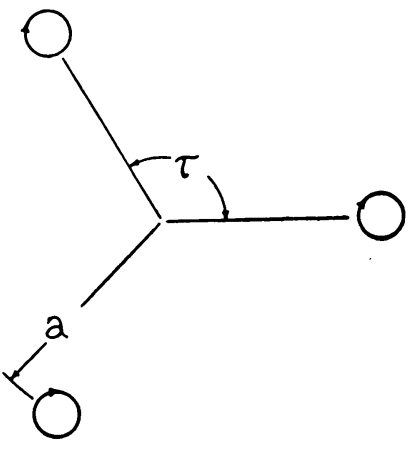

FIG. 1. Ring system of anticyclones.

In Table 1 are given the values of $\Omega$ and of $a \Omega \sqrt{g h_{0}}$, the linear velocity of the vortices,

\section{TABLE 1.}

Angular Velocities of Vortex Systems, $\alpha=0.06, N=3$

\begin{tabular}{c|c|c}
\hline \hline$a$ & $\Omega$ & $a \Omega \sqrt{g h_{0}}$ \\
\hline 3.0 & -.000112 & $-9.6 \mathrm{~cm} / \mathrm{sec}$ \\
3.5 & -.000037 & $-3.7 \mathrm{~cm} / \mathrm{sec}$ \\
\hline
\end{tabular}
for $\alpha=0.06, N=3$, and $a=3.0$ and 3.5. The values of $a=3.0$ and 3.5 correspond to the ring of subtropical anticyclones being placed at latitude $37^{\circ}$ and $29^{\circ}$ respectively. From this result it is seen that such a vortex system would have a slow precession to the west. In the atmosphere, there is also a region of distributed cyclonic vorticity to the north of the westerly winds. It is easily seen that this cyclonic vorticity tends to produce an eastward displacement of the subtropical highs. It appears that these two displacements cancel one another so that the systems are practically stationary, a condition which is undoubtedly also imposed by the thermodynamic and topographical factors. Since the westward drift shown in Table 1 is very small, no attempt will be made to correct the vortex system shown in Fig. 1 in order to take into account the polar cyclonic vorticity.

If the $n$th vortex is displaced by a distance $\Delta r_{n}$ in the radial direction and by an angle $\Delta \theta_{n}$ in the tangential direction, the surface deflection in the displaced condition is

$$
\eta=\alpha \sum_{n=1}^{N} K_{0}\left[\left(a+\Delta r_{n}\right)^{2}+r^{2}-2\left(a+\Delta r_{n}\right) r \cos \left(\theta-n \tau-\Delta \theta_{n}\right)\right]^{1 / 2} .
$$


The velocities of the vortices may be calculated as before by Eq. (9). If the displacements are small, the changes in velocity of the $N$ th vortex from the equilibrium value indicated by Eq. (10) may be written as

$$
\begin{aligned}
\Delta u_{r}= & \frac{d}{d t}\left(\Delta r_{N}\right)=\frac{\alpha}{2} \sum_{n=1}^{N-1} \Delta r_{n} K_{0}\left(R_{n}\right) \sin (n \tau) \\
& +\alpha \sum_{n=1}^{N-1} a\left(\Delta \theta_{n}-\Delta \theta_{N}\right)\left\{\frac{K_{1}\left(R_{n}\right)}{R_{n}}+K_{0}\left(R_{n}\right) \cos ^{2} \frac{1}{2} n \tau\right\} \\
\Delta u_{\theta}= & a \frac{d}{d t}\left(\Delta \theta_{N}\right)+\Omega \Delta r_{N}=\alpha \Delta r_{N} \sum_{n=1}^{N-1}\left\{K_{0}\left(R_{n}\right) \sin ^{2} \frac{1}{2} n \tau-\frac{K_{1}\left(R_{n}\right)}{R_{n}} \cos (n \tau)\right\} \\
& +\alpha \sum_{n=1}^{N-1}\left[\Delta r_{n}\left\{K_{0}\left(R_{n}\right) \sin ^{2} \frac{1}{2} n \tau+\frac{K_{1}\left(R_{n}\right)}{R_{n}}\right\}+\frac{1}{2} a \Delta \theta_{n} K_{0}\left(R_{n}\right) \sin (n \tau)\right],
\end{aligned}
$$

where

$$
R_{n}=2 a \sin \frac{1}{2} n \tau .
$$

It should be noted that the $t$ in Eq. (12) is a dimensionless time. If the actual time is $t^{*}$, then

$$
t=2 \omega t^{*} \text {. }
$$

Expressions similar to Eq. (12) for the velocities of the other vortices could be written from symmetry. These would form a set of simultaneous differential equations for the displacements.

If the $N$ equations in each of the two sets indicated in Eq. (12) are added, it is seen that

$$
\begin{gathered}
\frac{d}{d t}\left\{\sum_{n=1}^{N} \Delta r_{n}\right\}=0 \\
\frac{d}{d t}\left\{\sum_{n=1}^{N} \Delta \theta_{n}\right\}=\alpha\left\{\sum_{n=1}^{N} \Delta r_{n}\right\}\left\{\sum_{n=1}^{N-1}\left[2 K_{0}\left(R_{n}\right) \sin ^{2} \frac{1}{2} n \tau+\frac{1}{a} K_{1}\left(R_{n}\right) \sin \frac{1}{2} n \tau\right]\right\} .
\end{gathered}
$$

From this it is seen that if a mean value of $a$ is chosen so that $\sum_{n=1}^{N} \Delta r_{n}=0$ initially, then from Eq. (14) both $\sum_{n=1}^{N} \Delta r_{n}$ and $\sum_{n=1}^{N} \Delta \theta_{n}$ will remain constant. These results correspond to similar equations for two dimensional line vortices which state that the impulse of a system having no external forces remains constant. ${ }^{3}$

The disturbed motion of the vortex system as described in Eq. (12) can best be discussed by considering the normal modes of oscillation. From the symmetry conditions, the displacements in each normal mode must be of the form $\Delta r_{n}=\Delta r_{N} e^{i n \varphi}$ and $\Delta \theta_{n}=\Delta \theta_{N} e^{i n \varphi}$ where $\varphi$ characterizes the normal mode and is a member of the series $2 \pi / N, 4 \pi / N, \cdots, 2 \pi(N-1) / N, 2 \pi$. With this notation, Eq. (12) can be written as"

$$
\frac{1}{\alpha} \frac{d}{d t}\left(\Delta r_{N}\right)=A \Delta r_{N}-B a \Delta \theta_{N}, \quad \frac{a}{\alpha} \frac{d}{d t}\left(\Delta \theta_{N}\right)=C \Delta r_{N}+A a \Delta \theta_{N},
$$

where

${ }^{3}$ Lamb, H., Hydrodynamics, 6th edition, Cambridge University Press, London, 1932, 220. 


$$
\begin{aligned}
A & =\frac{1}{2} \sum_{n=1}^{N-1} K_{0}\left(R_{n}\right) e^{i n \varphi} \sin (n \tau) \\
B & =\sum_{n=1}^{N-1}\left(1-e^{i n \varphi}\right)\left\{\frac{K_{1}\left(R_{n}\right)}{R_{n}}+K_{0}\left(R_{n}\right) \cos ^{2} \frac{1}{2} n \tau\right\} \\
C & =\sum_{n=1}^{N-1}\left\{K_{0}\left(R_{n}\right)\left(1+e^{i n \varphi}\right) \sin ^{2} \frac{1}{2} n \tau+\frac{K_{1}\left(R_{n}\right)}{R_{n}}\left(1-2 \cos (n \tau)+e^{i n \varphi}\right)\right\} .
\end{aligned}
$$

If the amplitudes $\Delta r_{N}$ and $\Delta \theta_{N}$ are assumed to vary like $e^{i p t}$ and $p$ is the dimensionless normal frequency, then from Eq. (15),

$$
p=\alpha\{-i A \pm \sqrt{B C}\} .
$$

From Eq. (16), it can be seen that for $\varphi=2 \pi, A=B=0$ and there are thus two zero normal frequencies. These two zero frequencies are those shown in Eq. (14). It can also be seen that for the specified values of $\varphi, A$ is always a purely imaginary quantity, $B$ is always real and not less than zero. From Eq. (17), the condition that the frequencies be real is that $C$ be real and non-negative. Complex frequencies, of course, characterize systems in which the amplitudes increase with time and are thus unstable. Now $C$ is always real and is always positive for $N<7$. If $N=7, C$ is always positive if $a>71$. For $N>7, C$ is negative for one or more of the given values of $\varphi$. A value of $a>71$ corresponds either to disturbances of such great wave lengths or to motions of such a shallow layer of air in the earth's atmosphere that it probably is of no significance. The vortex system is thus stable if $N$ is less than or equal to six.

The frequencies and modes of oscillation will be discussed in some detail for the cases where $N=3$ and $a=3.0$ and 3.5. From Eq. (13) it may be seen that the period of an oscillation is given by

$$
T=\left|\frac{1}{2 p}\right| \text { sidereal days. }
$$

The normal mode of oscillation, from Eq. (15), is given by

$$
\frac{a \Delta \theta_{n}}{\Delta r_{n}}=\frac{a \Delta \theta_{N}}{\Delta r_{N}}=\frac{A-i p / \alpha}{B} .
$$

The results for $a=3.0$ and 3.5 are given in Tables 2 and 3, respectively. The two normal modes thus show a short period ( 2000 days to 5000 days) and a long period oscillation (70,000 days to 250,000 days). The path of the vortex is in each case an ellipse. For the short period oscillation the vortices are east of their mean position when traveling south and west when traveling north. For the long period oscillation the sense of the rotation is reversed.

Conclusion. The present calculations cannot be considered as a quantitative theory of the oscillations of the semi-permanent high pressure systems; they must be considered rather as an existence proof. Since the essential features of the model, vorticity concentrations at distances of roughly $10,000 \mathrm{~km}$., are also found in the atmosphere, motions of this type must exist in the atmosphere. It might be expected that the effects of coupling between the systems of the Northern and Southern Hemispheres and of any cyclonic vorticity concentrations on the polar sides of the westerly winds 
TABLE 2.

Normal Modes of Oscillation FOR $N=3, a=3.0, \alpha=0.06$.

\begin{tabular}{|c|c|c|c|c|}
\hline$\varphi$ & \multicolumn{2}{|c|}{$2 \pi / 3$} & \multicolumn{2}{|c|}{$4 \pi / 3$} \\
\hline$-i A$ & \multicolumn{2}{|c|}{0.002248} & \multicolumn{2}{|c|}{-0.002248} \\
\hline$B$ & \multicolumn{2}{|c|}{0.00414} & \multicolumn{2}{|c|}{0.00414} \\
\hline$C$ & \multicolumn{2}{|c|}{0.001182} & \multicolumn{2}{|c|}{0.001182} \\
\hline$p$ & 0.000268 & 0.000002 & -0.000268 & -0.000002 \\
\hline$T$-days & 1,865 & $2.5 \times 10^{5}$ & 1,865 & $2.5 \times 10^{5}$ \\
\hline$\frac{a \Delta \theta_{N}}{\Delta r_{N}}$ & $-0.53 i$ & $+0.53 i$ & $+0.53 i$ & $-0.53 i$ \\
\hline
\end{tabular}

TABLE 3.

Normal Modes of Oscillation for $N=3, a=3.5, \alpha=0.06$.

\begin{tabular}{|c|c|c|c|c|}
\hline$\varphi$ & \multicolumn{2}{|c|}{$2 \pi / 3$} & \multicolumn{2}{|c|}{$4 \pi / 3$} \\
\hline$-i A$ & \multicolumn{2}{|c|}{0.000859} & \multicolumn{2}{|c|}{-0.000859} \\
\hline$B$ & \multicolumn{2}{|c|}{0.001467} & \multicolumn{2}{|c|}{0.001467} \\
\hline$C$ & \multicolumn{2}{|c|}{0.000375} & \multicolumn{2}{|c|}{0.000375} \\
\hline$p$ & 0.000096 & 0.000007 & -0.000096 & -0.000007 \\
\hline$T$-days & 5,200 & $7.1 \times 10^{4}$ & 5,200 & $7.1 \times 10^{4}$ \\
\hline$\frac{a \Delta \theta_{N}}{\Delta r_{N}}$ & $-0.51 i$ & $0.51 i$ & $0.51 i$ & $-0.51 i$ \\
\hline
\end{tabular}

would be to decrease the period of the shortest oscillation and to introduce additional natural frequencies. No attempt has as yet been made to estimate the magnitude of these effects or of the errors involved in using velocity distributions corresponding to vortices on a rotating disc rather than to vortices on a rotating sphere and in neglecting the seasonal variations in the strength of the semi-permanent high pressure systems. It is suggested that the present calculations may prove useful as a guide for the statistical analysis of empirically obtained data. 